\title{
Trends and Progress in Collaborative Robot Applications
}

\author{
Saša Sladić, Robert Lesjak \\ Faculty of Engineering, University of Rijeka \\ Vukovarska 58 \\ 51000 Rijeka, Croatia \\ sladics@riteh.hr, rlesjak@riteh.hr
}

\author{
Lidija Runko Luttenberger, Mateja Šnajdar Musa \\ School of Polytechnics, University of Rijeka \\ Sveučilišna avenija 4 \\ 51000 Rijeka, Croatia \\ lidija.luttenberger@uniri.hr, mateja.snajdar@uniri.hr
}

\begin{abstract}
Modern robots have been used in different applications including welding, painting, soldering, assembly of different products and in education. List of applications is getting even longer because robot performance is improving. Faculties are following industries involving robots in their curriculum, while industry is interested in new ideas including new applications and their improvements from the university. Modern robots are userfriendly for programming so the lack of knowledge about robot applications seems to be the main obstacle in their widerimplementation. Collaborative robots or cobots are sophisticated robots which could operate with other robots and with workers in the factory. Recent opening of Yaskawa factory in Kočevje, Slovenia, near border with Croatia, has significant impact on integrating the robots in production and education in central and South-east Europe.
\end{abstract}

Keywords: industrial revolutions; collaborative robots; cobot applications; teaching; education.

\section{Introduction}

Recent trends in industry could be described with the term Industry 4.0. This term is targeted to mechatronics and fully automated factories which could be controlled by a single person. Such factory is automated; however, data collecting is more intensive than in earlier factories. Robots are designed in such a way that they could operate together (with ea ch other) or with humans. This type of cyber-physical systems makes a smart factory. Any fault in smart factory is indicated immediately including material and fuel shortages or similar. Basic element of such sophisticated system is a collaborative robot, or abbreviated, cobot. The applications of cobots are increasing, and future workers and engineers will operate with them. That is a reason why proper education in this field is important (Piedade, 2020). Res earch in this subject shows that limitation factor in collaborative robot applications arises from the lack of knowledge or experience in referent cases and potential applications (Aaltonen, Salmi, 2019), (Weitian at all, 2019). In other hand robot programming is important because of pattern recognition, programming, abstract and critical thinking, debugging and designing systems. Therefore, this paper deals with importance of collaborative robot applications and their improvement.

\section{Industrial revolutions and trends in industry and society}

Now, in the third decade of the $21^{\text {st }}$ century human technologyandsocial development throughout the last 200 years could be res umed as being framed by four industrial revolutions. First industrial revolution 
is connected to the a pplications of stea m engine (Fig. 1). The first industrial revolution brings advances to humanity in the form of more reliable and costeffective traveling across larger distances with simultaneous increase in production. The second industrial revolution started more than 100 years ago, with mass production of Ford T model. In the following years a utomobiles became affordable to many households making the strong impact to the American culture and mobility, at the same time supporting economy around the world. The third industrial revolution relates to the automation in industry and application of microcomputers. Electronics gave significant impact to the third industrial revolution since personal computers and their industrial counterparts PLC (programmable logic controllers) appeared and have started to develop in following decades. That time was important in regard to power electronics since thyristors were present in industry enabling the robust electronic control of power. It should be mentioned that robots that took part in the third industrial revolution appeared in a simple form.

\begin{tabular}{|l|l|l|l|}
\hline $\begin{array}{l}\text { 1. Industrial revolution } \\
\text { Steam engine, } \\
\text { water power }\end{array}$ & $\begin{array}{l}\text { 2. Industrial revolution } \\
\text { automobile mass production }\end{array}$ & $\begin{array}{l}\text { 3. Industrial revolution } \\
\text { PLCs, simple robot } \\
\text { applications, thyristor control }\end{array}$ & $\begin{array}{l}\text { 4. Industrial revolution } \\
\text { Mechatronic systems, Internet } \\
\text { of Things, 3D printing }\end{array}$ \\
\hline Around year: 1850 & Year: 1910 & Around year: 1970 & Year: 2020 \\
\hline
\end{tabular}

Figure 1. Four industrial revolutions

Thus, fourth industrial revolution is underway in 21 century ( $\varnothing$ stergaard, 2017). It is propelled by many sophisticated sys tems including their mutual interaction. These sophisticated systems include collaborative robots or cobots, internet of things (IOT), 3D printers, artificial intelligence and other advanced technologies. Such approach generated changes of automated factories previously run by humans.

For example, robotized factory could operate in the dark, since programmed robots do not need daylight, and the light is turned on only when the humans enter the production area. Similar issue is regarding factory windows; robots do not need windows thus many robotized factories have been built without windows. Such factories are run by microcontrollers and microcontrollers are produced in such production systems, since semiconductor industry is dominantly robotized.

Generally, fourth industrial revolution is the term used to describe late improvement in technologies involved in production process. Booth machining and semiconductor production should be considered, along with social change, connected with the influence of powerful microcontrollers (e.g. ARM cortex cores) applied in mobile phones, TV receivers, cameras, washing machines, automobiles and el sewhere gave their contribution. Nowadays every home has 50 and more microcontroll ers in different devices. This number is still increasing. Internet of things gives a nother meaning to home appliances. Air conditioning could be controlled remotely, similar as preparing a hot water. The renewables should also be included in a single a utoma ted system known as smart home. Smart factory assumes even more sophisticated systems giving us the possibility to observe social changes as compared to earlier life.

\section{Collaborative robots}

Res ea rch shows that limitation factor in collaborative robots or cobots seem to be lack of knowledge or experience about referent cases and potential applications (Aaltonen, Salmi, 2019). Workers could not operate with a tra ditional industry robot because of their physical barrier used as a protection from fast-moving robotic arms. During the last decade, a 
new class of robots has a ppeared. This class of robots could operate with humans in shared environment which is called collaboration (Matheson, 2019). Robot programming has undergone significant development recently, so today's collaborative robots a re user-friendly to program and flexible to adapt to different applications. Collaboration of humans and robots could lead to cost effective solutions in manufacturing processes. Absence of additional safety equipment contributes to flexibility and low costs. Collaboration with other collaborative robots is possible and furthermore collaboration between traditional and collaborative robots has been investigated.

According to literature (Matheson, 2019) five (for some authors four) types of humans to robots' interactions are possible.

1. First type of human/robot interaction is that where there basically is no interaction. In roboticcells, robots are divided by mechanical fence or other protection approaches due to security reasons. This type of interaction has been defined as robotic cell with no interaction between human and robot (Fig. 2).

2. Second type of robot/human interactions is coexistence. This operation mode does not involve anyinteraction with working a rea of both human and a robot divided, lacking mechanical fence.

3. Third type of interaction has been called synchronism. In this operation mode both robot and a humanoperate in the same environment but not at the same time. Interaction is avoided by time scheduling of certain operation.

4. Fourth type of interaction between humans and robots is called cooperation. This operation mode combines operating environment of human and robot, each one is doing his/its own task.

5. Fifth type of interaction between a human and a robot has been called collaboration. In this case human and robot are working together performing the same task. This is possible by means of force sensors and vision systems used by collaborative robot (Weitian at all, 2019), (Sabatino Scimmi, 2021).

Most a dvanced interaction between a human and a robot involves collaborative robots or cobots. This is most advanced case in human robot interaction which enables a wide possibility of applications for collaborative robots however, other cases are also important since collaborative robots are slower in operation. Market demands shows collaborative robot increase ever since their appearance (Matheson, 2019) few years ago, especially in education (Piedade, 2020), (Aaltonen, Salmi, 2019), (Lopez-Caudana, 2020).

\section{Cobots applications}

The use of cobots can obtain high value in industries where the production process changes rapidly and productionis in small to medium scale, thus human work is required. In such scenarios developing and building specialized tooling does not make sense. Another push forward with cobot applications are the new production lines worldwide which influence in their cost effectiveness.

Opening of the new factoryin Slovenia, near the border with Croatia, offers a potential for significant monthly production of robots and increase of the number of robots used in local factories. However, local schools and faculties (Fig. 3) also take part in approaching technological changes by educating their students in the sense that they could run, service and improvelocal factories with implemented cobot labor force.

Collaborative robots have additional sensors which means that robotic hand movement will be stopped in the case of contact with human or another robot.

In this manner robot does not operate in cage (robotic cell) but is rather positioned near to human (U. Robots, 2018). This new paradigm enables different applications of robots which are yet to be discovered.

Basically, cobot could be programmed in three ways: a) using robot pendant (Fig. 1) where human operator moves the robotic arms in order to perform different tasks; b) using CAD programs for programming a robot and; $\mathrm{c}$ ) teaching the robot by the human (Fig. 3), which means memorizing different points in s pace to program the robotic arm movement, whereby teaching could be categorized in different sub methods of tea ching (Weitian at all, 2019), (Ren, 2019), (Lee, 2009).

The methods of robot learning available in literature include robot vision and precise force sensors which enable robots to be more flexible in different applications. Examples from the automotive industry show benefits of using cobots (Heydaryan, Suaza Bedolla, Belingardi, 2018) where a human-robot interaction is needed. This application involves human hands flexibility and robot reliability during long lasting repetitive operations. Another reports state that collaboration between robots is of high importance in cases of potential disasters occurrence (Grigore, 2020). For instance, people cannot operate under high radiation, so cobots have to work together. Lately, camera applications are more often used in cobots operation (Amin, 2020). 


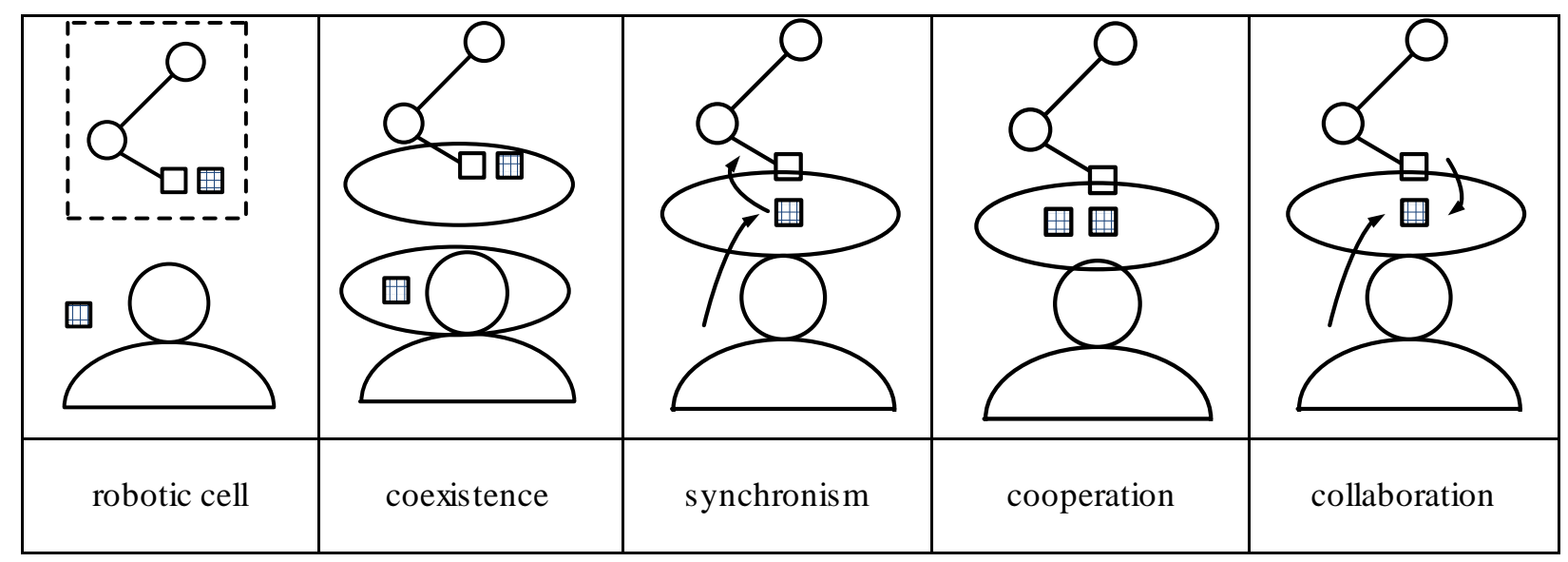

Figure 2. Interaction types between a robot and a human
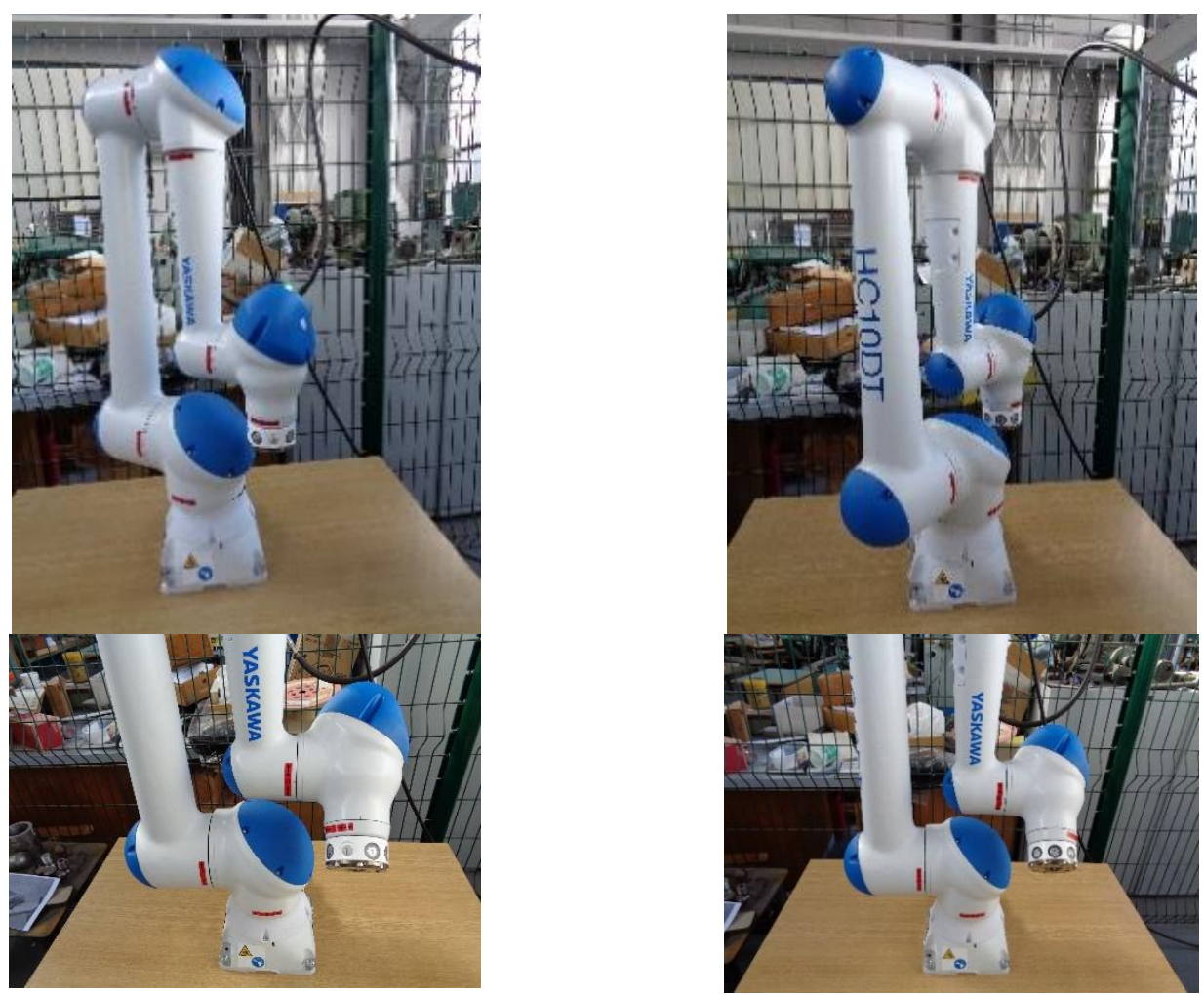

Figure 3. Collaborative robot (cobot) HC10DT from Yaskawa used in teaching process (temporarily positioned on wooden desk), Faculty of Engineering, University of Rijeka, Croatia 
That means the collaboration could be better implemented through object recognition and artificial intelligence applications. From the educational point of view robotic applications have positive effect on different fields of human thinking and development. In study (Piedade, 2020) was mentioned graphical programming, however in case of YRC1000 which has been used for control of Yaskawa HC10DT (Fig.3) a standard high-level programming language based on $C$ has been used. Even more important is system design and abstract thinking which should be applied in order to imagine robot movement in frame of computer program with different conditional statements like while, for, if else statements known from the $\mathrm{C}$ and other programming languages because robot motions should be repea ted in frame of different coordinates. It is important to mention significance of robot collaboration in educational purposes because in this way students could operate directly on robot without mechanical fence. During the robot teaching process and cobot moti on loop defi nition one of three speeds could be selected. Selected points could be connected by shortest line or rounding during the motion should be applied.

Increasing number of applications results in higher demand for robots, especially cobots. Such demand for increased cobot applications in industry pushes robot producers to increase their production. For example, Yaskawa in 2018 opened four new factories in three countries: China, Japan and Slovenia (Yaskawa report, 2018). Electric drives are closelyrelated to robots so Yaskawa has expressed plans to open factory for electric motors and to introduce more cobots in that segment of production with the support of highly-trained engineers.

\section{Conclusion}

Society in general is greatly influenced by all industrial revolution phases ranging from steam engine to collaborative robots as ma in representative of modern cyber-physical systems. Modern production areas are adjusted both to robot operation and to collaborative robot/humans' operation. These plants are automated and production data are intensively collected. Modern universities should prepare their students to get us ed to the work with cobots, devel op new applications in the framework of different production processes and to improve existing production processes. Programming knowledge directed primarily to production could be used for other services, e.g. serviceactivities in restaurants such is cooking or customer servicing. Furthermore, robot servicing demands high levels of electronics understanding, therefore making it a vital part of university education programs, resulting in new generations of highly-skilled engineers.

\section{References}

Aaltonen, I., Salmi, T.: Experiences and expectations of collaborative robots in industry and a cademia: barriers and development needs, 29th International Conference on Flexible Automation and Intel ligent Manufacturing (FAIM2019), June 24-28, 2019, Limerick, Ireland, Procedia Manufacturing 38 (2019) 1151-1158

Amin, F. M., Rezayati, M., Wernher van de Venn, H., Karimpour, H.: AMixed-Perception Approach for Safe Human-Robot Collaboration in Industrial Automation, Sensors 2020, 20, 6347; doi:10.3390/s20216347

Grigore, L. S., Priescu, I., Joita, D., Oncioiu, I.: The Integration of Collaborative Robot Systems and Their Environmental Impacts, Processes 2020, 8, 494; doi :10.3390/pr8040494

Heydaryan, S., Bedolla, J. S., Belingardi, G.: Safety Design and Development of a Human-Robot Collaboration Assembly Process in the Automotive Industry, Appl. Sci. 2018, 8, 344; doi:10.3390/app8030344

Lee, Li, C., Kim, D., Kyung, J., Han, C.: THE DIRECT TEACHING AND PLAYBACK METHOD FOR ROBOTIC DEBURRING SYSTEM USING THE ADAPTIVEFORCE-CONTROL, Proceedings of 2009 IEEE International Symposi um on Assembly and Manufacturing 17-20 November 2009, Suwon, Korea

Lopez-Caudana, E., Soledad Ramirez-Montoya, M., Martínez-Pérez, S., Rodríguez-Abitia, G.: Using Robotics to Enhance Active Learning in Mathematics: A Multi-Scenario Study, Mathematics 2020, 8, 2163; doi:10.3390/math8122163

Matheson, E., Minto, R., Zampieri, E. G. G., Faccio, M., Rosati, G.: Human-Robot Collaboration in Ma nufacturing Applications: A Review, Robotics 2019, 8, 100; doi:10.3390/robotics 8040100

$\emptyset$ stergaard, E. H.: THE ROLE OF COBOTS IN INDUSTRY 4.0, Universal Robots, white paper, 2017.

Piedade, J., Dorotea, N., Pedro, A., Matos, J. F.: On Teaching Programming Fundamentals and Computational Thinking with Educational Robotics: ADidactic Experience with Pre-Service 
Teachers, mdpi, Educ. Sci. 2020, 10, 214; doi:10.3390/educs ci100902DTP]

Ren, T., Dong, Y., Wu, D., Chen, K.: Design of Direct Teaching Behavior of Collaborative Robot Based on Force Interaction, J Intel I Robot Syst 96, 83-93 (2019). https://doi.org/10.1007/s 10846-01900986-3

Scimmi, L. S., Melchiorre, M., Troise, M., Mauro, S., Pastorelli, S.: A Practical and Effective Layout for a Safe Human-Robot Collaborative Assembly Task, Appl. Sci. 2021, 11, 1763. https://doi.org/10.3390/app11041763

U. Robots, An introduction to common colla bora tive robot applications, White Pap. (2018).

Wang, W., Chen, Y., Li, R., Jia, Y.: Learning and Comfort in Human-Robot Interaction: A Review, Appl.Sci. 2018, 8, 344; doi:10.3390/app8030344, Appl.Sci. 2019, 9, 5152; doi:10.3390/app9235152

Yaskawa report 2018. https://www.yaskawaglobal.com/wpcontent/uploads/2018/08/ar2018_E-27-30.pdf 